\title{
Ginsenoside Rg2 Attenuates Ischemia/Reperfusion- induced Injury to Spinal Cord in Rats
}

\author{
X. YU, MEI XIN¹, A. HUANG, L. CHEN ${ }^{1}$, X. CAI, J. SHEN ${ }^{1}$, T. ZOU, T. YANG ${ }^{1}$ AND Y. MIAO* \\ Department of Orthopedics, Suzhou Municipal Hospital, The Affiliated Suzhou Hospital of Nanjing Medical University, \\ ${ }^{1}$ Department of Orthopedics, The First Affiliated Hospital of Soochow University, Suzhou, P. R. China
}

Yu et al.: Ginsenoside Rg2 Attenuates Ischemia-induced Spinal Cord Injury in Rats

\begin{abstract}
To investigate the pathological mechanism underlying the injury caused by ischemia/reperfusion and reperfusion and explore the role of ginsenoside $\mathrm{Rg} 2$ on ischemia/reperfusion injury to spinal cord, 24 male Sprague Dawley rats underwent a surgical procedure in this study. The rats were divided into 3 groups, group 1- sham operated group $(n=8)$, group 2- surgical ischemia/reperfusion $(n=8)$ and group 3- surgical ischemia/reperfusion+ginsenoside $\mathrm{Rg} 2(\mathrm{n}=8)$. Oxidative stress, apoptosis and pathological indices were observed if any. Thirty minutes of ischemia, reduced the lumbar spinal cord blood flow by $77.48 \%$ of the baseline and as the reperfusion was initiated, local blood flow increased rapidly and exceed the baseline level, and continued to below the levels of baseline later. However, it returns to baseline levels local blood perfusion after reperfusion $1 \mathrm{~h}$. Last for $3 \mathrm{~h}$ then it drops to $207.13 \% \pm 38.25 \mathrm{RU}$, but the value of blood flow not recovery. In addition, the level of oxidative stress increased in a time-dependent manner after ischemia/ reperfusion. Ginsenoside Rg2 could significantly reduce the ischemia/reperfusion-induced oxidative stress and apoptotic injury to the spinal cord in the rat. These results demonstrated that pathological mechanism changes during ischemia/reperfusion injury and with reperfusion time correlation and compliance. This study also confirmed that ginsenoside Rg2 could inhibit ischemia/reperfusion-induced oxidative stress and apoptosis in the rat spinal cord, thus providing a new insight for the treatment for ischemia/reperfusion injury of the spinal cord in future.
\end{abstract}

Key words: High ginsenoside Rg2, spinal cord injury, I/R injury, oxidative stress, apoptosis

Spinal cord injury caused by occlusion of the thoraco-abdominal aorta remains an unpredictable and devastating complication of aortic surgery for the resection of thoraco-abdominal aneurysms ${ }^{[1]}$. Individuals paralyzed by trauma to the spinal cord are left with one of the most physically disabling and psychologically devastating conditions known to humans. Most of the injured patients are under $30 \mathrm{y}$ old and the cost of operation and treatment is high, which brings serious economic burden to family and society $^{[2,3]}$. Spinal cord ischemia reperfusion injury (SCIR) is a devastating complication of thoracoabdominal aortic surgery ${ }^{[4,5]}$ and can be occasionally seen after surgical or endovascular treatment of spinal vascular lesions. In the central nervous system, any serious decrease in the blood flow causes energy failure and triggers a cascade of events leading to oxidative stress and apoptotic damage through amplification of many pathways set in motion by ischemia ${ }^{[6]}$.

Ginseng regarded as the king of Chinese herbal medicine, has been used in clinical practice of traditional
Chinese medicine for more than $2000 \mathrm{y}^{[7]}$. Ginsenoside Rg2 is a main active component of ginseng, which has significant therapeutic effect in many diseases ${ }^{[8]}$. Previous studies have shown that ginsenoside $\mathrm{Rg} 2$ can significantly inhibit oxidative stress and apoptosis ${ }^{[9]}$, but its effect on SCIR injury has not been fully elucidated so far. In this study, in vivo ischemia/reperfusion (I/R) injury model of spinal cord was established in rats. The role of ginsenoside $\mathrm{Rg} 2$ in I/R-induced oxidative stress and apoptosis was also explored to provide a scientific basis for the treatment and utilization of ginsenoside Rg2 in SCIR injury.

Rg2 (purity $>95 \%$ ) was a product from Jiangsu Yongjian Pharmaceutical Technology Co (Jiangsu, China). It was dissolved in dimethyl sulfoxide to obtain stock solution for further investigation. A total of 24 male Sprague Dawley (SD) rats were randomly divided into 3 groups, the normal group, the I/R group and the $\mathrm{I} / \mathrm{R}+$ ginsenoside $\mathrm{Rg} 2$ group. The control group received sham operation. In the $\mathrm{I} / \mathrm{R}$ group, $30 \mathrm{~min}$ after spinal cord injury, blood perfusion was restored. 
In the $\mathrm{I} / \mathrm{R}+$ ginsenoside $\mathrm{Rg} 2$ group, ginsenoside $\mathrm{Rg} 2$ pretreatment was given 2 weeks before I/R operation, and $10 \mathrm{mg} / \mathrm{kg}$ of ginsenoside $\mathrm{Rg} 2$ was fed every day. Total protein of spinal cord tissue was extracted and lysed in lysis buffer (Sigma, USA). Sample containing $30 \mu \mathrm{g}$ protein was exposed to $12 \%$ SDS-PAGE gels and transferred to nitrocellulose membranes. Primary antibody of Bax and bcl-2 was used in experiment and glyceraldehyde-3-phosphate dehydrogenase was used as a loading control. To evaluate oxidative stress level after I/R injury, blood from the 3 groups was assayed for the reactive oxygen species (ROS) and malondialdehyde (MDA) using methods that have been described previously ${ }^{[10]}$. Histopathologic evaluations were performed by a pathologist blinded to the study ${ }^{[11]}$. Histopathologic sections were evaluated both qualitatively and quantitatively in order to maximize scientific accuracy. Histologic damage was scored qualitatively, using a previously defined system to explain the pathological mechanism underlying the SCIR injury. All data were expressed as mean \pm SD. One-way Analysis of variance (ANOVA, followed by the Bonferroni test was used for multiple comparisons using GraphPad Prism 6.0 and $\mathrm{p}<0.05$ was considered statistically significant.

As some injuries to the spinal cord cause the osteoligamentous spinal column to fail under a variety of loading conditions, including flexion, extension, axial load, rotation and distraction. These forces impart the primary mechanical insult to the spinal cord, which in its mildest form causes a cord concussion with brief transient neurologic defects and in its most severe form causes complete and permanent paralysis. Whereas the former represents local axonal depolarization and transient dysfunction, the latter represents a primary axonal and neuronal injury followed by a spreading of secondary tissue damage that expands from the injury epicenter, the schematic and detailed explanation of which is shown in fig. 1 . The primary and secondary damage after spinal cord injury have played an important role in the mechanism of injury.

The dynamic variation of spinal cord blood perfusion (SCBP) is shown in Table 1. The baseline value of SCBP was $303.5 \pm 45.76 \mathrm{RU}$, the initial SCBP during ischemia dropped rapidly and when global ischemia maintained for $10 \mathrm{~min}$, SCBP dropped to $38.66 \pm 17.92 \mathrm{RU}$, which is $73.98 \%$ of the baseline. After $30 \mathrm{~min}$ ischemia, the local lumbar SCBP rapidly dropped to $77.48 \%$ of the baseline and as the reperfusion is initiated, local blood flow increased rapidly and exceeded the baseline level but stabilized at below the levels of baseline later. However, it returned to baseline levels after $1 \mathrm{~h}$ reperfusion. After $3 \mathrm{~h}$ reperfusion the blood flow maintained at $207.13 \pm 46.14 \mathrm{RU}$, which was lower than

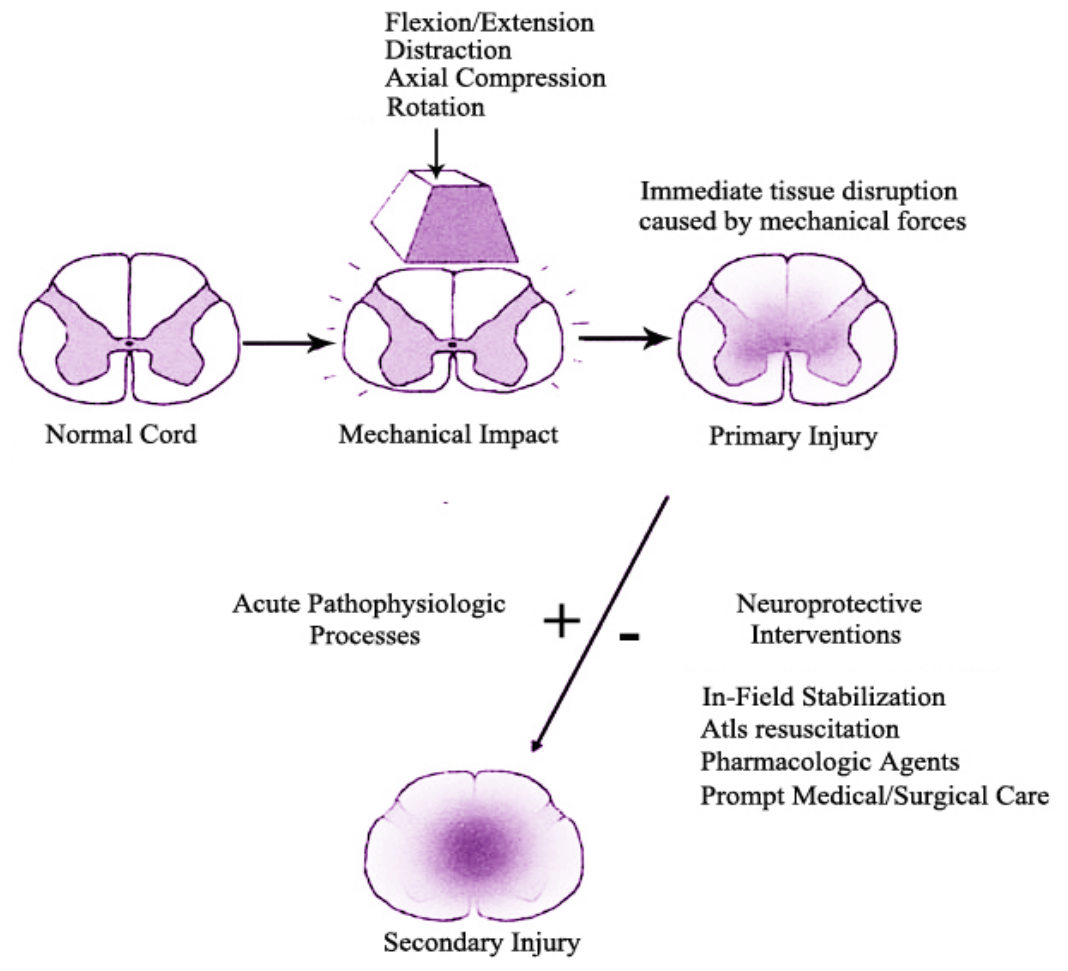

Fig. 1: Schematic presentation of primary and secondary damage after spinal cord injury Primary and secondary damage after spinal cord injury. The mechanical forces imparted to the spinal cord at the time of injury cause immediate tissue disruption 
the baseline. I/R injury could significantly increase the level of oxidative stress and free radical production in vivo. However, there were few studies which reported varying oxidative stress and its trend at different time points of I/R. In this study 10 and $30 \mathrm{~min}$ of ischemia, 30 , $60,120,180$ and $240 \mathrm{~min}$ of reperfusion were chosen to detect ROS and MDA levels. Results showed that $10 \mathrm{~min}$ of ischemia did not induce significant oxidative stress, but $30 \mathrm{~min}$ of ischemia led to a significant increase in oxidative stress. After reperfusion, oxidative stress increased in a time-dependent manner but after $3 \mathrm{~h}$ of reperfusion, oxidative stress index did not increase any further (fig. 2).

Fig. 3 shows the histopathologic changes in the anterior horn of lumbar spinal cord segments procured hours after reperfusion. The features representing neuronal injury that included eosinophilic cytoplasm, vacuolization and pyknotic appearance of nuclear structure. Cells that contained Nissl substance in the cytoplasm, loose chromatin and prominent nucleoli were considered viable. The viability index was calculated as the number of clearly viable neurons divided by the total neuronal count within the anterior horn of each section for each animal. Analysis of the tissue presented in fig. 3 is as follows, red arrows suggested inflammatory cell accumulation, destruction and vacuolization of the gray matter, pyknosis of neurons and capillary proliferation. To investigate the role of $\mathrm{Rg} 2$ in SCIR oxidative stress and apoptosis in vivo, western blot analysis was performed. It was observed that $2 \mathrm{~h}$ reperfusion after ischemia could significantly induce ROS, MDA level and the apoptotic protein Bax/Bcl2 ratio, while could be significantly inhibited by $\mathrm{Rg} 2$ (fig. 4).

The secondary injury that followed primary spinal cord injury during SCIR is an important factor that caused

\section{TABLE 1: LUMBAR SPINAL CORD BLOOD FLOW DURING ISCHEMIA AND REPERFUSION}

\begin{tabular}{lcc}
\hline Time $(\min )$ & SCBP $(\mathrm{RU})$ & Percent baseline \\
\hline Baseline & $303.5 \pm 45.76(30)$ & $-\cdots-\cdot-\cdot$ \\
I 10 & $38.66 \pm 17.92(5)$ & -73.98 \\
I 30 & $42.19 \pm 13.86(5)$ & -77.48 \\
R 10 & $427.09 \pm 88.37(4)^{* *}$ & 37.17 \\
R 30 & $326.92 \pm 71.33(4)$ & 18.05 \\
R 50 & $249.57 \pm 72(4)$ & -17.84 \\
R 60 & $244.31 \pm 29.17(4)^{* *}$ & -19.27 \\
R 90 & $246.35 \pm 52.29(4)$ & -20.57 \\
R 120 & $239.87 \pm 53.18(4)$ & $-22.87 \%$ \\
R 180 & $207.13 \% \pm 46.14(3)$ & $-33.91 \%$ \\
\hline
\end{tabular}

$\mathrm{RU}$ is the unit of blood reperfusion, Baseline is the value before ischemia, $I$ is ischemia and $R$ is reperfusion, compare to baseline value: ${ }^{* *} \mathrm{p}<0.01$

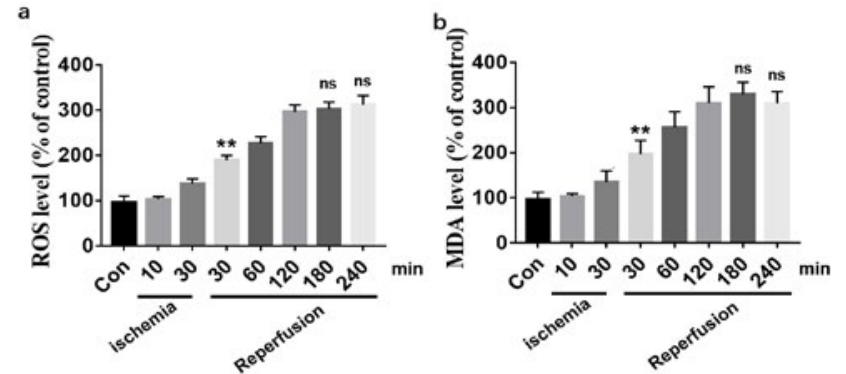

Fig. 2: ROS and MDA levels at different time points of ischemia and reperfusion

Reactive oxygen species (ROS) and malondialdehyde (MDA) levels at different time points of $I / R$ pathological process. a. ROS level. b. MDA level. ** $\mathbf{P}<0.01$ vs con; $n s$ means no significance
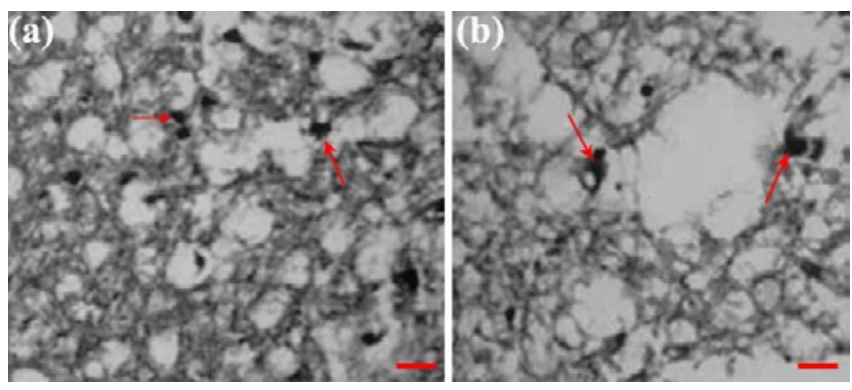

Fig. 3: Histopathology of anterior horn of lumbar spinal cord Histopathologic observation of anterior horn of lumbar spinal cord segments procured hours after reperfusion, (a) $1 \mathrm{~h}$, (b) 3 h, Scar bar $=100 \mu \mathrm{M}$

nerve cell injury ${ }^{[12]}$. After some factors leading to spinal cord ischemia were removed blood supply to spinal cord was restored, but the nerve function instead of getting improved was further aggravated, which led to delayed irreversible death of spinal cord neurons ${ }^{[13]}$. In addition, prolonged observations indicated that the local SCBP changes correlated well with pathological findings. Ischemic ultra-structural changes of spinal cord anterior horn cells after reperfusion were further aggravated and with prolonged reperfusion, spinal cord ventral horn cells also developed high degree of swelling, ambiguous nuclear envelope structure, intracytoplasmic vacuoles and increased cohesion of mitochondria, while under ischemia and subsequent hypoxia myelinated nerve fibers demonstrated dissolution and capillary endothelium thickened. Micro-villi type hairs appeared projecting in to endovascular structures resulting in endothelial injury as a consequence to the SCIR, which caused dual damage with increasing importance to the pathological basis of primary injury.

The SCIR model used in this study was first reported by Lang-Lazdunski et al. ${ }^{[14]}$. According to that report, the SCIR injury model in rats was developed, in which blood perfusion was observed at different time points during the pathogenesis of $\mathrm{I} / \mathrm{R}$ injury, as well as the 


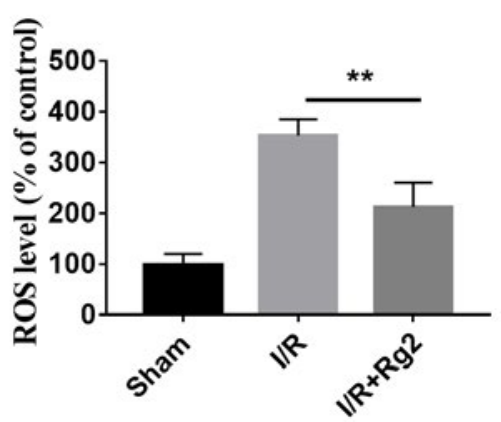

b

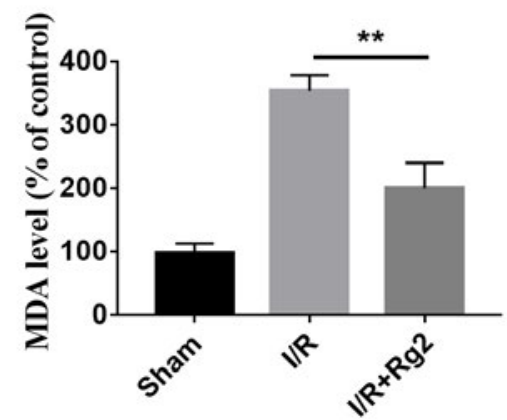

c

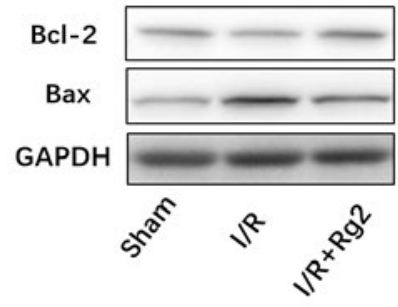

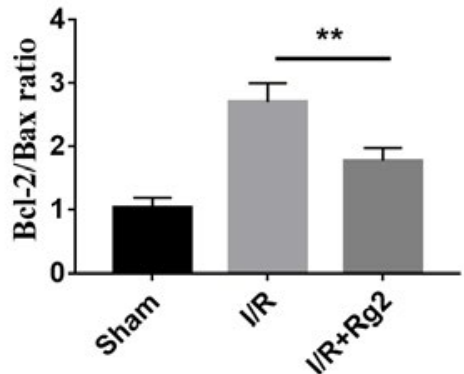

Fig. 4: Effect of ginsenoside Rg2 on SCIR-induced oxidative stress and apoptosis in rat

Rg2 inhibited spinal cord ischemia reperfusion (SCIR)-induced oxidative stress and apoptosis in rat. a-b. ROS and MDA level increased by $\mathrm{I} / \mathrm{R}$ damage was blocked by $\mathrm{Rg} 2$. c. Bcl-2/Bax ratio elevated by $\mathrm{I} / \mathrm{R}$ was decreased by $\mathbf{R g} 2 .{ }^{*} * \mathbf{P}<0.01$

levels of ROS and MDA were determined at different time points. It was found that after $30 \mathrm{~min}$ ischemia, the local blood flow of lumbar spinal cord rapidly dropped to $77.48 \%$ of the basal blood flow. Upon reperfusion initially local blood flow increased rapidly well above the basal level, but continued to remain below the basal level with prolonged reperfusion. However, it returned to basal level of local blood perfusion after reperfusion for $1 \mathrm{~h}$. Three hour reperfusion resulted in new basal blood flow level of $207.13 \pm 38.25 \mathrm{RU}$, which was actually lower than the basal level. Moreover, the level of oxidative stress increased in a time-dependent manner after I/R injury and peaked at $120 \mathrm{~min}$.

Ginsenoside, the main bioactive component of ginseng, has been successfully isolated and more than 50 ginsenoside monomers were identified, including ginsenoside $\mathrm{Rb} 1, \mathrm{Rb} 2, \mathrm{Rg} 2, \mathrm{RC}$ and $\mathrm{Re}^{[8]}$. Like other ginsenoside monomers, $\mathrm{Rg} 2$ appear to possess a wide range of biological functions, such as antioxidant, antiinflammatory, antitumor and immunoregulatory ${ }^{[15]}$. In this study, it was found that ginsenoside $\mathrm{Rg} 2$ significantly inhibited oxidative stress and apoptosis induced by SCIR injury. In conclusion, the present study explored blood flow and oxidative stress levels at different time periods after I/R. The study also found that ginsenoside $\operatorname{Rg} 2$ could significantly reduce the level of I/R-induced oxidative stress and apoptosis. These results provide new insights for the use of ginseng in the treatment of SCIR injury.

\section{Conflict of Interest:}

Authors do not have any conflicts of interest.

\section{REFERENCES}

1. Lukacova N. Ischemia-reperfusion injury in the spinal cord of rabbits strongly enhances lipid peroxidation and modifies phospholipid profiles. Neurochem Res 1996;21:869-73.

2. Tator $\mathrm{CH}$, Koyanagi I. Vascular mechanisms in the pathophysiology of human spinal cord injury. J Neurosurg 1997;86:483-92.

3. Harrop JS, Sharan AD, Vaccaro AR, Przybylski GJ. The cause of neurologic deterioration after acute cervical spinal cord injury. Spine 2001;26:340-6.

4. Kaptanoglu E, Okutan O, Akbiyik F, Solaroglu I, Kilinc A, Beskonakli E. Correlation of injury severity and tissue Evans blue content, lipid peroxidation and clinical evaluation in acute spinal cord injury in rats. J Clin Neurosci 2004;11:879-85.

5. Genovese T, Mazzon E, Crisafulli C, Di Paola R, Muià C, Bramanti P. Immunomodulatory effects of etanercept in an experimental model of spinal cord injury. J Pharmacol Exp Ther 2006;316:1006-16.

6. Gris D, Marsh DR, Oatway MA, Chen Y, Hamilton EF, Dekaban GA, et al. Transient blockade of the CD11d/CD18 integrin reduces secondary damage after spinal cord injury, improving sensory, autonomic, and motor function. J Neurosci 2004;24:4043-51.

7. Liu, Y, Fan D. Ginsenoside Rg5 induces apoptosis and autophagy via the inhibition of the PI3K/Akt pathway against breast cancer in a mouse model. Food Funct 2018;9:5513-27.

8. Li TJ, Lian ML, Yu D, Shao CH, Piao XC. Effects of several factors on cell growth and ginsenoside accumulation of Panax ginseng suspension culture. Chin Materia Med 2013;38:404751.

9. Cho YL, Hur SM, Kim JY, Kim JH, Lee DK, Choe J, et al. 
Specific activation of insulin-like growth factor-1 receptor by ginsenoside $\mathrm{Rg} 5$ promotes angiogenesis and vasorelaxation. J Biol Chem 2015;290:467-77.

10. Ma Y, Wang D, Xu X, Yang X, Wang X, Zhu Z, et al. Dynamic changes of ROS, MDA and SOD during arsenic-induced neoplastic transformation in human keratinocytes. Wei Sheng Yan Jiu 2015;44:456-61.

11. Toumpoulis IK. Resveratrol or higher arterial blood pressure protects the spinal cord from ischemia-reperfusion injury? Ann Thorac Surg 2006;82:1572-3.

12. Abe Y, Yamamoto T, Sugiyama Y, Watanabe T, Saito N, Kayama $\mathrm{H}$, et al. Apoptotic cells associated with wallerian degeneration after experimental spinal cord injury. A possible mechanism of oligodendroglial death. J Neurotrauma 1999;16:945-52.

13. Lin R, Roseborough G, Dong Y, Williams GM, Wei C. DNA damage and repair system in spinal cord ischemia. J Vasc Surg 2003;37:847-58.
14. Lang-Lazdunski L, Matsushita K, Hirt L, Waeber C, Vonsattel JP, Moskowitz MA. Spinal cord ischemia: Development of a model in the mouse. Stroke 2000;31:208-13.

15. Zhang QL, Li SY, Li P. Effects of ginsenoside-Rg on mechanical allodynia, heat hyperalgeia, depressive state of rats with chronic sciatic nerve constriction injury. Chin J Appl Phys 2019;35:228-31.

This is an open access article distributed under the terms of the Creative Commons Attribution-NonCommercial-ShareAlike 3.0 License, which allows others to remix, tweak, and build upon the work non-commercially, as long as the author is credited and the new creations are licensed under the identical terms

This article was originally published in a special issue, "Recent Trends in Biomedical Research"

Indian J Pharm Sci 2020:82(1)spl issue1; XX-XX 